\title{
GÖTTERBILDER IM RÖMISCHEN GRIECHENLAND: VOM TEMPEL ZUM MUSEUM?
}

\author{
Christoph Auffarth
}

\section{Vom Tempel zum Museum: Fragestellung und Begrenzung der Frage ${ }^{1}$}

Cicero trat als Anwalt der Göttin Ceres auf, als er den Kunstraub des Verres und insbesondere den Raub ihrer Statue aus Henna anprangerte. Wie konnte sich der römische Gouverneur anmaßen, eine Göttin aus ihrem Tempel zu rauben? ${ }^{2}$ Wenn man aber sieht, in welchem Maßstab in der römischen Kaiserzeit der Kunsthandel mit griechischen Statuen aufgezogen wurde, und wie leicht aus einem Götterbild das Meisterwerk eines bestimmten Künstlers werden konnte, das sich nach dem ästhetischen Wert des Kunstwerks bemaß und nicht nach der Bedeutung der dargestellten Gottheit oder der Zahl ihrer Verehrer, so drängt sich der Eindruck auf, dass die Ästhetik die Religion ersetzte - oder, etwas schwächer gesagt, eine Ästhetisierung der klassischen Religion einsetzte, die auch für die römische Religion eine Veränderung der Maßstäbe zur Folge hat. Hieraus kann man folgendes Postulat entwickeln: Aus dem Mutterland der klassischen Religion, der römischen Provinz Achaia, wurde eine Sammlung

${ }^{1}$ Dieser Aufsatz ist Teil einer größeren Arbeit und fügt sich ein in eine Reihe weiterer Studien des Verfassers, die einiges Material und Quellen zum Teil ausführlicher vorstellen und diskutieren: C. Auffarth, 'Das angemessene Bild Gottes: Der Olympische Zeus, antike Bildkonvention und die Christologie', in: N. Kreutz - B. Schweizer (Hrsg.), Tekmeria. Archäologische Zeugnisse in ihrer kulturhistorischen und politischen Dimension. Beiträge für Werner Gauer (Münster 2006), 1-23; ders., 'Teure Ideologie - billige Praxis: Die „kleinen“ Opfer in der römischen Kaiserzeit', in: E. Stavrianopoulou - A. Michaels - C. Ambos (Hrsg.), Transformations in Sacrificial Practices: From Antiquity to Modern Times (Berlin 2008), 147-170 (= Auffarth 2008a); ders., 'Religio migrans. Die ,Orientalischen Religionen' im Kontext antiker Religion. Ein theoretisches Modell', in: C. Bonnet - S. Ribichini - J. Rüpke (Hrsg.), Religioni in contatto nel mondo antico. Modalità di diffusione e processi di interferenza (Rom 2008), 291-321 (= Auffarth 2008b); ders., 'Reichsreligion und Weltreligion', in: J. Rüpke (Hrsg.), Die Religion des Imperium Romanum. Koine und Konfrontation (Tübingen 2008), 37-53.

${ }^{2}$ Cicero, In Verrem II 4, 106-115. 
künstlerischer Meisterwerke; das aes Corinthium wurde zu einem Schlagwort, das den illegalen Handel mit kleineren, beweglichen Kunstwerken, darunter religiösen Paraphernalien, bezeichnete. ${ }^{3}$ Kurz gesagt: Das alte Griechenland wurde zum Museum.

Man kann durchaus Argumente finden, die diese Sichtweise bestärken. Aber man verstellt sich dadurch den Blick auf die Veränderungen der klassischen Religion, die eben nicht das einfache Ersetzen einer rituellen Struktur durch Ästhetik bedeuteten, sondern eine Metamorphose der Religion. Gerade an den Ritualen lässt sich das beobachten. Und dass dabei die gewandelten Machtstrukturen eine Zentralisierung religiöser Angelegenheiten zur Folge haben, wird an den behandelten Gegenständen deutlich werden. ${ }^{4}$

Mit den Götterbildern und deren Einbindung in den Kult verbinden sich zum einen neu aufgeworfene Fragen, insbesondere für den griechischen Bereich, zum anderen die Herausforderung, dass gerade beim Kultbild - neben dem Tieropfer - der härteste Bruch zwischen den antiken ikonischen Religionen und dem anikonischen Christentum gegeben zu sein scheint. Die späte Einführung von Bildern in den Kult der Christen hatte eine rituelle Seite, an der neben Differenzierung auch Übernahme und Kontinuität erkennbar ist. ${ }^{5}$ Magisches ,survival' oder ,Hellenisierung des Christentums' sind theologische Modelle, die religionswissenschaftlich keine Alternativen und erst recht keine ausreichenden Kategorien sind. Die Debatte darüber, was das visuelle Medium für die Repräsentation des Stifters von Ritualen und damit die religiöse Praxis bedeutet, wird zwar von Intellektuellen geführt, aber sie betrifft jeden Kultteilnehmer, sobald die Hand-

\footnotetext{
${ }^{3}$ M. Pape, Griechische Kunstwerke aus Kriegsbeute und ihre öffentliche Aufstellung in Rom. Von der Eroberung von Syrakus bis in augusteische Zeit (Diss. Hamburg 1975); G. Hellenkemper Salies (Hrsg.), Das Wrack. Der antike Schiffsfund von Mahdia I-II (Köln 1994). Immer noch grundlegend für die literarische Seite der Rezeption: H. Jucker, Vom Verhältnis der Römer zur bildenden Kunst der Griechen (Frankfurt a. M. 1950); R. Neudecker, Die Skulpturenausstattung römischer Villen in Italien (Mainz 1988); T. Hölscher, 'Hellenistische Kunst und römische Aristokratie', in: Hellenkemper Salies 1994 II, 875-888.

${ }^{4}$ Eine Definition der nicht zentralisierten, nicht an die Machtausübung gebundenen ,Reichsreligion' (eher: ,Religion im Römischen Reich') findet sich bei Auffarth 2008b, a.a.O. (Anm. 1); ders. im Druck, a.a.O. (Anm. 1).

${ }^{5}$ Dies geschah im Zusammenhang mit der Entwicklung einer Bildertheologie nach dem Einbruch des Islam und dem Ikonoklasmus im östlichen, dann auch im westlichen Mittelmeer, insbesondere durch Johannes von Damaskos. Vgl. D.J. Olewiński, Um die Ehre des Bildes. Theologische Motive der Bilderverteidigung bei Johannes von Damaskus (St. Ottilien 2004), bes. 580-586.
} 
lung nicht als ,selbstverständlich ‘ angesehen wird. Der Diskurs um das angemessene Bild ist weit älter als die Diskussion zwischen den Intellektuellen der beiden ,Philosophien', der klassischen und der christlichen. Um verstehen zu können, was am Ende mit der Entwicklung des christlichen Verehrungsbildes geschaffen wurde, muss ich (kursorisch) einige Aspekte der klassischen Götterbilder ansprechen.

\section{Das Ende der griechischen Religion}

Das scheinbare Ende der griechischen Religion wird häufig folgendermaßen rekonstruiert: Mit dem Einfall der Heruler in Griechenland sei die griechische Religion im Jahre 267 unserer Zeitrechnung zu Ende gegangen. Diese These will ich zunächst am Beispiel von Olympia diskutieren, um weiter zu fragen, welche Veränderung die griechische Religion unter dem Einfluss der römischen Herrschaft durchgemacht hat. Was ist in und nach der Krise des römischen Reiches im 3. Jahrhundert aus der griechischen Religion geworden? Dabei ist natürlich auch die Frage zu berücksichtigen, welche Rolle in der religiösen Landschaft die Herausforderung durch das prinzipiell so unterschiedliche Christentum spielte.

Zunächst also der Blick auf Olympia: In den Darstellungen zum Ende Olympias wurde der Einfall der Heruler im Jahr 267 als dasjenige Ereignis angesehen, das zur Einstellung der Spiele und der Kulte geführt habe; das Erdbeben von 290 habe dann alle Aktivitäten vollends zunichte gemacht. Alfred MALLWITZ etwa schrieb noch 1988: „So gesehen war Olympia schon rund 130 Jahre vor dem Verdikt Theodosius' I. tot". ${ }^{6}$ Das Verbot der Olympischen Spiele und der damit verbundenen griechischen Kulte sprach Kaiser Theodosius I. im Jahr 394 aus. Demnach müsste man dies als ein Für-TotErklären und Beerdigen der Leiche eines vor langer Zeit Verstorbenen bewerten. In der genannten Zeit, also in der Mitte des 3. Jhs., wurden der Zeustempel und die Altis - wohl in Erwartung des Ein-

${ }^{6}$ A. Mallwitz, Olympia und seine Bauten (München 1972), 313 lässt in einer Graphik mit dem Titel „Lebensdauer der Bauwerke“ fast alle Kulte mit dem Jahr 267 enden. Das Zitat stammt aus aus A. Mallwitz, 'Olympia in der Spätantike', Antike Welt 19/2 (1988), 21-45, hier 43. 
falles der Heruler ${ }^{7}$ - in eine Festung einbezogen, die sich südlich davon erstreckte und auch die Echohalle einschloss (Abb. 1). Die wertvollen Statuen aus der Altis wurden in den so gebildeten Innenhof der Festung zwischen die südlichen Säulen des Zeustempels gebracht und eingedübelt (Abb. 2) Auf den ersten Blick passt dieser Vorgang $\mathrm{zu}$ den Thesen von der Ästhetisierung und Musealisierung, um nicht zu sagen Mumifizierung, der griechischen Religion - aus dem Tempel scheint ein Museum geworden zu sein. Der Ausgräber MALLWITZ schloss hieraus, dass in der Zeit danach in Olympia kein aktiver Kult mehr betrieben wurde.

Diese Annahme haben nun aber neue Befunde aus Olympia eindeutig widerlegt: Zum einen ist eine Inschrift gefunden worden, die Sieger in den Olympischen Spielen noch aus der Mitte des 4. Jhs. nennt, also bis kurz vor dem kaiserlichen Verbot. ${ }^{8}$ Zum andern lässt sich die Geschichte Olympias in der Spätantike nun genauer rekonstruieren. ${ }^{9}$ Die dauerhafte städtische Siedlung im Süden der Altis zeigt, dass hier bis zur slawischen Migration im Übergang zum Mittelalter kontinuierlich Menschen wohnten. Aus den beiden neuen Befunden lässt sich erschließen, dass in Olympia ,das Fest' durch Einflüsse von außen beendet wurde und dies durch ein generelles Verbot heidnischer Gottesdienste verschärft werden musste. Der schönen Formel, dass die Christen die Statuen und Kultbilder geschützt hätten, indem sie sie säkularisiert und ästhetisiert hätten - darauf deuten unter anderem kaiserliche Erlasse zum Schutz der Kunstwerke hin ${ }^{10}$

\footnotetext{
${ }^{7}$ Skeptisch zum Realitätsgehalt des Heruler-Einfalles äußern sich U. Sinn, 'Der griechische Tempel: Kulisse für den Altar', in: N. Bock et al. (Hrsg.), Kunst und Liturgie im Mittelalter (München 2000), 55-64; S. Lehmann, 'Der ,Herulersturm' und die Kunstproduktion in der Provinz Achaia', in: E. Walde - B. Kainrath (Hrsg.), Die Selbstdarstellung der römischen Gesellschaft in den Provinzen im Spiegel der Steindenkmäler. IX. Internationales Kolloquium über Probleme des Provinzialrömischen Kunstschaffens, Innsbruck 2005 (Innsbruck 2007), 45-54; A. Gutsfeld - S. Lehmann, Olympia in der Spätantike (Tübingen 2009), im Druck.

8 J. Ebert, 'Zur neuen Bronzeplatte mit Siegerinschriften aus Olympia Inv. 1148', Stadion 24/1 (1998), 217-234.

${ }^{9}$ Vgl. Gutsfeld - Lehmann im Druck, a.a.O. (Anm. 7) sowie das Forschungsprojekt der beiden Autoren zu den Panhellenischen Heiligtümern in der Spätantike. Dies. - J. Hahn, ,Christlicher Staat und ,panhellenische' Heiligtümer', in: J.Rüpke (Hrsg.), Antike Religionsgeschichte in räumlicher Perspektive, Tübingen 2007, 228 237.

${ }^{10}$ Dazu H.-R. Meier, 'Alte Tempel - neue Kulte. Zum Schutz obsoleter Sakralbauten in der Spätantike und zur Adaption alter Bauten an den christlichen Kult', in: B. Brenk (Hrsg.), Innovation in der Spätantike (Wiesbaden 1996), 361-374; O. Dal-
} 
- stehen Befunde gegenüber, die auf die gewaltsame Schließung und Zerstörung von heidnischen Gottesdiensträumen hinweisen. Chronologische Konstruktionen, denen zufolge die Griechen die Tempel langsam aufgegeben hätten und erst geraume Zeit später Christen in die verlassenen Gebäude eingezogen seien, erscheinen daher fragwürdig. ${ }^{11}$ Die gewaltsame Zerstörung des Serapeions von Alexandria ist somit nicht mehr als Einzelfall zu bewerten, ${ }^{12}$ denn das Verbot Theodosius' I. ist nicht der Schlussstrich unter ein bereits beendetes Kapitel antiker Religion gewesen, sondern es war ein staatlicher Gewaltakt, der aber nicht überall gleichzeitig, sondern mit Verzögerungen realisiert wurde. Die Forschungen zur spätantiken Phase in den Panhellenischen Heiligtümern zeigen deutlich, dass dort auch nach 392 weiterhin Kult ausgeübt wurde.

Mithin starb die griechische Religion nicht in der Reichskrise des dritten Jahrhunderts oder im konstantinischen Zeitalter, sondern sie durchlief eine tiefgreifende Metamorphose, die bereits vorher eingesetzt hatte und nicht (oder zumindest nicht allein) durch die Konfrontation mit einer neuen Religion zu erklären ist. Es geht hierbei im Wesentlichen um den Vorgang der Universalisierung durch Ästhetisierung.

\section{Das Ende des Opfers}

Das Phänomen der Ästhetisierung ist die eine Seite eines komplementären Vorgangs, auf dessen anderer Seite der Rückgang des Rituals steht. Hier muss man allerdings noch einmal genauer differen-

ly, 'Pflege und Umnutzung heidnischer Tempel in der Spätantike', in: G. Brands H.-G. Severin (Hrsg.), Die spätantike Stadt und ihre Christianisierung (Wiesbaden 2003), 97-114.

${ }^{11}$ Vgl. R.M. Rothaus, Corinth, the First City of Greece. An Urban History of Late Antique Cult and Religion (Leiden 2000), 32-63. Rothaus argumentiert gegen A. Frantz, 'From Paganism to Christianity in the Temples of Athens', Dumbarton Oaks Papers 19 (1965), 185-205 und F.W. Deichmann, 'Frühchristliche Kirchen in antiken Heiligtümern', Jahrbuch des Deutschen Archäologischen Instituts 54 (1939), 105-136. Dagegen schon J.M. Spieser, 'La christianisation des sanctuaires paiens en Grèce', in: H. Kyrieleis (Hrsg.), Neue Forschungen in griechischen Heiligtümern (Tübingen 1976), 309-320; R.R. Trombley, Hellenic Religion and Christianization, c. 370-529(Leiden 1995, 2. Aufl.) I, 283-332; II, 377-379.

${ }^{12} \mathrm{~J}$. Hahn, Gewalt und religiöser Konflikt. Studien zu den Auseinandersetzungen zwischen Christen, Heiden und Juden im Osten des Römischen Reiches von Konstantin bis Theodosius II. (Berlin 2004), 81-94. 
zieren. Denn vor allem ein Ritual schwand und wurde schließlich gesetzlich geächtet - das blutige Opfer. Das blutige Opfer war ein ideologisch hoch angesehenes Ritual, dessen reale Bedeutung gegenüber den anderen, ,kleinen' Formen des Opfers aber meist überschätzt wird. ${ }^{13}$ Die Restitution des Opferrituals in der kurzen Herrschaft Julians, des sogenannten Apostaten, hätte, glaubt man den gängigen Theorien über das blutige Opfer, als Wiederherstellung des zentralen Rituals der antiken Religionen Begeisterung hervorrufen müssen. Im Gegenteil aber stieß die Erneuerung der Opfer überall im Imperium Romanum auf Ablehnung. Das blutige Opfer passte einfach nicht mehr zu den Vorstellungen der Zeitgenossen und der ästhetischen Würde der Religion. Schon vor dem Verbot der christlichen Kaiser galt das blutige Tieropfer als altmodisch oder gar abstoßend, und bereits die heidnischen Herrscher hatten entsprechende Verordnungen erlassen, freilich mit einer anderen Begründung. ${ }^{14}$

Julian selbst kam aus einer Tradition und führte diese weiter, die weniger in rituellen Handlungen als in hymnischen Preisungen, die man mit anderen Gelehrten teilte, Religion realisierte - eine philosophische Religion des Wortes, eine ,Intellektuellenreligion'. ${ }^{15}$ Man verband sie jedoch mit konkreten Götterpersonen aus dem traditionellen Kult und nahm an dessen Ritualen teil, so dass letztere nicht durch Sublimation in die Intellektuellenreligion aufgehoben wurden. Die Rückkehr zum barbarisch-blutigen Opfer lehnten die meisten Befürworter der philosophischen Religion allerdings ab. Das ,Ende des Opfers' stellt sich somit dar als eine Beschränkung des großen, blutigen Opferns auf rituelle Formen, während die ,kleinen Opfer die kultische Handlung ausmachten. Das hat erhebliche Konsequenzen, deren Ausführung den Rahmen dieses Aufsatzes sprengen würde und an anderer Stelle teils schon dargelegt sind, teils aber noch zu erörtern sind. ${ }^{16}$

${ }^{13}$ Auffarth 2008a, a.a.O. (Anm. 1); Trombley 1995, a.a.O. (wie Anm. 11) I, 197.

${ }^{14}$ Vgl. neben Auffarth 2008a, a.a.O. (Anm. 1), vor allem N. Belayche, 'Sacrifice and the theory of sacrifice during the ,pagan reaction': Julian the Emperor', in: A.I. Baumgarten (Hrsg.), Sacrifice in Religious Experience (Leiden u.a. 2002), 101-126.

${ }^{15}$ I. Tanaseanu-Döbler, Konversion zur Philosophie in der Spätantike. Kaiser Julian und Synesios von Kyrene (Stuttgart 2008); dies., 'Befreiung aus der Finsternis: Kaiser Julian und die orientalischen Kulte', in: Bonnet - Ribichini - Rüpke 2008, a.a.O. (Anm. 1), 281-301.

${ }^{16}$ Einen wichtigen Aspekt des Themas behandelt G.G. Stroumsa, La fin du sacrifice. Les mutations religieuses de l'antiquité tardive (Paris 2005). 


\section{Der Tempel als Museum}

Schon seit längerem ist die Frage nach der Funktion des Tempels insbesondere in der griechischen Welt zu einem Rätsel geworden. Die Verbindung zum Altar ist an vielen Stellen zwar augenscheinlich, aber es gibt genügend Beispiele, bei denen der Altar für sich steht und daher das Ritual ohne Bezug zum Tempel ausgeführt worden sein muss. Das Beispiel des Pelops-Altars in Olympia, der sich in seiner Lage weder dem Hera- noch dem Zeus-Tempel zuordnen lässt, ist ein Beispiel dafür. So sind Ideen entwickelt worden, den Tempel als Votivstiftung anzusehen oder in manchen Fällen als Siegesmonument zu verstehen.

Besonders prägnant stellt sich das Interpreationsproblem aber in Bezug auf die Götterbilder: Wenn das Bild der Gottheit im Opferritual in der Regel nicht einbezogen wurde (die griechischen Götter nehmen die Fleischmahlzeit ja durch die Nase auf), so ergibt sich unweigerlich die Frage, welche Rolle das Bild im Kult überhaupt gespielt hat. Der Begriff des ,Kultbildes' überdeckt die hier aufscheinenden Probleme, ${ }^{17}$ denn es muss zunächst geklärt werden, in welcher Beziehung das Bild zum Ritual stand. ${ }^{18}$ Ein wichtiger Hinweis lässt sich aus dem Phänomen der Verdoppelung des Bildes gewin-

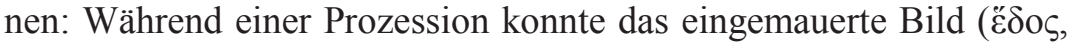

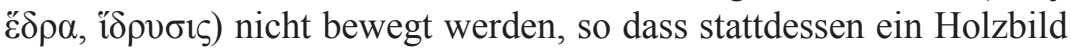
umhergetragen wurde. Ansonsten blieb letzteres aber eher im Hintergrund, während das große, aus besonders wertvollem Material gefertigte ,Bild der Bewunderung' im Zentrum des Interesses stand. Dass den Griechen die Differenz zwischen der Gottheit und ihrem

${ }^{17}$ Eine klare Unterscheidung trifft T. Scheer, Die Gottheit und ihr Bild. Untersuchungen zur Funktion griechischer Kultbilder in Religion und Politik (München 2000). Wenig problembewusst ist hingegen G. Nick, Die Athena Parthenos. Studien zum griechischen Kultbild und seiner Rezeption (Mainz 2002).

${ }^{18}$ Wichtiges Material findet sich jetzt in: Thesaurus Cultus et Rituum Antiquorum II: Purification, Initiation, Heroization, Apotheosis, Banquet, Dance, Music, Cult Images (Los Angeles 2004), bes. 417-507 zur physischen Behandlung der Kultbilder, zum Waschen, Kleiden, Ernähren, Bekränzen und zur Verehrung der lebenden Bilder, Prozessionen etc.; sowie zur Vorstellung, Kultbilder seien vom Himmel gefallen oder nicht von Menschenhand gemacht (acheiropoietoi), und zur Zerstörung von Kultbildern. Vgl. außerdem Thesaurus Cultus et Rituum Antiquorum IV: Cult Places, Representations of Cult Places (Los Angeles 2005), 52-65. Rezension hierzu: C. Auffarth, Numen 55 (2008), 101-104. 
Abbild bewusst war, ist in den Quellen klar zu sehen. ${ }^{19}$ Jedenfalls kam es nicht zu der Anbetung eines materiellen Bildes, wie es die intellektuelle (auch christliche) Polemik später darstellte.

Um seiner Anklage Nachdruck zu verleihen, hebt Cicero im Kontrast zum Kunstraub des Verres die Göttlichkeit des Kultbildes hervor, dessen Macht als Reaktion auf seine Entehrung die Menschen in Form von Missernten geschädigt habe (Cicero, In Verrem II 4, 114). Aber die Betonung der lebendigen Macht des Bildes ist der rhetorischen Funktion dieser Stelle geschuldet. Dass Ästhetisierung nicht im Widerspruch zur Einbindung eines Bildes in den Kult stand, zeigt eine Stelle bei Pausanias: Das Kultbild des Apollon von Bassai wird von den Bürgern der Stadt Phigaleia an Megalopolis geschenkt, zur Verschönerung der neu gegründeten Stadt, und dort offen, d.h. ohne Integration in einen Tempel, aufgestellt. Vor dieser Statue wird ein Eber geopfert, von dem man dann Teile zu dem Apollonheiligtum weit draußen vor der Stadt trägt und dort für Apollon Parrhasios den Götteranteil verbrennt, während der Rest des Ebers den Prozessionsteilnehmern als eine Art als Spanferkel-Essen mundet. ${ }^{20}$ Dies geschah bereits in spätklassischer Zeit (370/69 v. Chr.) und macht deutlich, wie die Aspekte ,Kultbild' und ,künstlerisches Meisterwerk' zusammenfallen konnten. Dies ist jedoch nicht als Säkularisierung eines heiligen, als gotterfüllt gedachten Gegenstandes zu verstehen.

Der Tempel konnte auch - als Raum der Andacht - der Entfaltung einer innerlichen Religiosität des Individuums in der Beziehung $\mathrm{zu}$ seinem Gott dienen, wofür sich durchaus Beispiele finden lassen. Wenn aber Ulrich VON WILAMOWITZ-MOELLENDORF eine Gottesvision für das Musterbeispiel des „Glaubens der Hellenen“ gehalten hat, nämlich jene Epiphanie, die Sophokles um 420 v. Chr. zur Einführung des Asklepios-Kults in Athen bewegt haben soll, ${ }^{21}$ dann saß

${ }^{19}$ Auffarth 2006, a.a.O. (Anm. 1). Ein anderes Bild diskutiere ich ausführlicher in Auffarth 2008b, a.a.O. (Anm. 1).

${ }^{20}$ Pausanias 8.30.3 f.; 38.8; 41.8 f. Vgl. hierzu Fernande Hölscher, in: Thesaurus Cultus et Rituum Antiquorum IV: Cult Places, Representations of Cult Places (Los Angeles 2005), 61 Nr. 24.

${ }^{21}$ Plutarch, Moralia 1103A. Neuplatonisch überhöht findet sich dies in der Vita des Proklos 29 des Marinos. Die Interpretation als gelungene Vereinigung von individuellem Ergriffensein und dem „Glauben der Väter" bei U. von WilamowitzMoellendorff, Der Glaube der Hellenen II (Berlin 1932), 235. Zur Auffassung der griechischen Religion als ,Glaube' siehe C. Auffarth, 'Ein Gesamtbild der antiken Kultur. Adolf Erman und das Berliner Modell einer Kulturwissenschaft der Antike 
er einer späteren Interpretation auf. Dieser wichtige religionshistorische Vorgang beruhte offenbar nicht auf einer Vision, denn erst in der Kaiserzeit stellte Plutarch die persönliche Vision des Sophokles als das zentrale Ereignis für die Epiphanie des Asklepios in Athen dar. ${ }^{22}$ Und erst im 5. Jh. n. Chr. wurde daraus die neuplatonische Epiphanie.

Für die Metapher des Tempels, mit der der individuelle Körper eines Menschen als Wohnung Gottes bezeichnet wurde, lässt sich zeigen, dass sie nicht im Sinne einer Verdrängung bzw. als Abwendung vom rituellen Kult gemeint war, sondern dass beides nebeneinander und in harmonisierender Weise praktiziert werden konnte. Weder Paulus noch Seneca bringen das Bild vom Körper als Tempel gegen die realen Tempel in Jerusalem oder auf dem Kapitol vor. ${ }^{23}$

Mit all dem hängt die Diskussion darüber zusammen, welche Funktion das Götterbild im Zusammenhang mit dem Kult gehabt haben soll. „Der Tempel als Kulisse“ ist Ulrich SINNs Lösung: Der Tempel glich demnach einer Abstellkammer für Weihungen, einem „Kuriositätenkabinett“" oder einem völlig überfüllten Antiquitätenladen, wo auch Zähne eines Nilpferdes oder Straußeneier zu bewundern waren. ${ }^{24}$ Sicher waren dies keine Kultobjekte, aber Zeichen der Bedeutung des Heiligtums und seiner Gottheit im Weltmaßstab. Ein Museum im positiven Sinne repräsentiert ja die Identität einer lokalen Gruppe; in diesem Sinne ist ,der Tempel als Museum` auch Ort der Traditionspflege. ${ }^{25}$

um die Jahrhundertwende 1900', in: B.U. Schipper (Hrsg.), Ägyptologie als Wissenschaft. Adolf Erman (1854-1927) in seiner Zeit (Berlin - New York 2006), 396-433.

${ }^{22}$ C. Auffarth, 'Aufnahme und Zurückweisung ,Neuer Götter' im spätklassischen Athen: Religion gegen die Krise, Religion in der Krise', in: W. Eder (Hrsg.), Die athenische Demokratie im 4. Jahrhundert v. Chr.: Vollendung oder Verfall einer Verfassungsform? (Stuttgart 1995), 345-347.

${ }^{23}$ C. Auffarth, "Euer Leib sei der Tempel des Herrn“. Religiöse Sprache bei Paulus', in: D. Elm-von der Osten - J. Rüpke - K. Waldner (Hrsg.), Texte als Medium und Reflexion von Religion im Römischen Reich (Stuttgart 2006), 63-80.

${ }^{24}$ Sinn 2000, a.a.O. (Anm. 7). Vgl. auch S. Aleshire, Asklepios at Athens (Amsterdam 1991), 41-46; J.N. Bremmer, Götter, Mythen und Heiligtümer im antiken Griechenland (Darmstadt 1996), 41.

${ }^{25}$ T. Scheer, 'Ein Museum griechischer, Frühgeschichte' im Apollontempel von Sikyon', Klio 78 (1996), 353-373. 


\section{5. Ästhetischer Kanon und Kontinuität antiker Religion unter christlichen Namen}

Als ästhetisches Meisterwerk wurde das Zeusbild in Olympia zum Maßstab für das angemessene Götterbild. ${ }^{26}$ Mit großer Skepsis ist eine Nachricht aus byzantinischer Zeit zu bewerten, nach der die Zeusstatue aus Olympia um das Jahr 420 nach Konstantinopel abtransportiert und dort in ein Museum gestellt, fünfzig Jahre später aber bei einem Brand zerstört worden sein soll. Der Autor (Erfinder?) dieser Nachricht, Kedrenos, berichtet ansonsten teilweise ganz abenteuerliche Dinge, etwa, dass die gesamte Ilias auf einer Schlangenhaut aufgeschrieben wurde. Aber auch ohne die Geschichten des Kedrenos war der Olympische Zeus bereits Maßstab für die angemessene Darstellung Gottes geworden. So folgte beispielsweise die Veränderung des ägyptischen Apis-Stieres in eine menschengestaltige Statue dem Vorbild des Zeus von Olympia (Abb. 3). Andererseits passt die Nachricht des Kedrenos durchaus zu der Situation am Anfang des 5. Jahrhunderts, als das Bild Christi innerhalb kurzer Zeit grundlegend neu gestaltet wurde und aus der Repräsentation der ,Friedenskindlichkeit' der bärtige, gesetzte Pantokrator wurde. ${ }^{27}$ Falls hinter der Nachricht des Kedrenos ein ernst zu nehmendes Wissen steht, könnte das Bild des Zeus, als es nach Konstantinopel entführt wurde, durchaus noch ein Kultbild gewesen sein. Erst 426 erließ Theodosius II. das Edikt, dass kein Kult mehr in den heidnischen Tempeln gehalten werden durfte. ${ }^{28}$

Das Verbot der antiken Religion war aber kein Verbot der antiken Kunst. So wurde die antike Kunst zum Träger antiker Religion, weil sie weitgehend mit religiösen Zeichen angefüllt war. Man kann einen hierzu komplementären Satz aufstellen: Antike Religion lebte weiter, teils als (so die christliche Lesart) säkularisiertes Zeichen, teils als christianisierte religiöse Handlung. Sie wurde unter neuen

\footnotetext{
${ }^{26}$ Vgl. Auffarth 2006, (Anm. 1), 1-23 mit einer Diskussion der einschlägigen Quellen.

${ }^{27} \mathrm{Zu}$ der Kontroverse zwischen M. Büchsel, Die Entstehung des Christusporträts. Bildarchäologie statt Bildhypnose (Mainz 2003) und H. Belting, Bild und Kult. Eine Geschichte des Bildes vor dem Zeitalter der Kunst (München 2004, 6. Aufl.) siehe Auffarth 2006, a.a.O. (Anm. 1).

${ }^{28}$ C. Mango - M. Vickers - E.D. Francis, 'The palace of Lausus at Constantinople and its collection of ancient statues', Journal of the History of Collections 4/1 (1992), 93 f. Eusebius, De vita Constantini 3.48.
} 
Namen ausgeführt und um einige Formen reduziert. Nach einer heftigen innerchristlichen Kontroverse um die ,Anbetung ' oder Zerstörung der Bilder kam es schließlich zur ,Bildtheologie', dem christlichen Gebrauch des zweidimensionalen Kultbildes als Ikone. ${ }^{29}$

Obwohl das Bildnis einen heidnischen Gott darstellte, wurde die Zeusstatue inmitten der ,allerchristlichsten' Stadt aufgestellt, und zwar im Palast des Lausos. Neben dem Gold-Elfenbein-Bild des Zeus aus Olympia konnte man dort auch die Aphrodite des Praxiteles aus Knidos, die Hera des Lysippos aus Samos und die Athene Lindia von Skyllis und Dipoinos bewundern; ${ }^{30}$ dazu eine Bibliothek der antiken Klassiker von 120.000 Büchern. Schönheit war also bewahrenswert, selbst wenn sie die klassischen Götter darstellte; klassische Literatur musste man tradieren, auch wenn sie von Göttern und antiker Religion handelte. Bevor der Palast im Jahre 475 einem Brand zum Opfer fiel, war er rund 50 Jahre lang das Weltwunder schlechthin in der christlich geprägten Stadt. Die Schönheit, Erhabenheit und Würde des Zeus von Olympia prägten somit den Geschmack der Zeit. Die Christen eroberten erst zu dieser Zeit, im späteren 5. Jahrhundert, den öffentlichen Raum der Städte und zeigten Monumentalität in ihrer Architektur. Im Rahmen einer durch und durch ikonischen Kultur erkennt man dabei eine komplementäre Entwicklung: Auf der einen Seite wurden die klassischen Götterbilder aus ihrem Sitz im Leben und dem Kontext des Kultes herausgerissen; eine Entwicklung, die allerdings schon im Hellenismus eingesetzt hatte und während der römischen Kaiserzeit in zahlreichen Beispielen zu fassen ist. Auf der anderen Seite benötigten die Christen, da sie nun die öffentlichen Orte für sich besetzen wollten, das Medium der Sichtbarkeit, also die Monumentalarchitektur und das großformatige Bild. Anders gesagt: Als das Kaiserhaus Christus zum Leit-Gott erwählte, benötigte es ein entsprechendes Bild der herrschenden Gottheit. Das Bild des majestätischen Gottes wurde für die Christen im Idealbild des Pantokrators manifest. Dadurch wurde nun aber nicht etwa Zeus zum Gegengott, etwa als Abbild des Teufels. Für letzteres verwendeten die Christen vielmehr das Bild einer Gottheit, welche schon im griechischen Pantheon ein Außenseiter und Durcheinan-

\footnotetext{
${ }^{29}$ M. Wallraff, 'Tendenzen zum Monotheismus als Kennzeichen der religiösen Kultur der Spätantike', Verkündigung und Forschung 52/2 (2007), 74 f.

${ }^{30}$ Für weitere Beispiele s. H. Funke, 'Götterbild', in: Reallexikon für Antike und Christentum XI (Stuttgart 1981), 739-741.
} 
derbringer war: Pan mit seinen Hörnern, dem Bocksfuß und dem Schwanz. ${ }^{31}$ Wie all dies mit der Nachricht von der gewaltsamen Schließung und Zerstörung des Tempels in Olympia zu verknüpfen ist, muss hypothetisch bleiben; immerhin ist hierfür das Datum 426 überliefert. Die Anknüpfung der Christologie vom Gott=Menschen an die klassisch-antike Theologie geschieht über die in der kaiserzeitlichen Kunsttheorie modellhaft anhand des Zeusbildes in Olympia entwickelte Vorstellung, als dessen Erschaffer Phidias zum Musterbeispiel für den schöpferischen Meister wurde. Im Unterschied zu den verachtenswerten sogenannten Künstlern, die Gott abbilden und ihn dabei durch die Verwendung von Stein oder Holz entwerten, können die schöpferischen Menschen geradezu das Göttliche im Holz oder Metall zum Leben bringen. Das Kultbild wird so zum Ort der Epiphanie, es markiert den Ort der möglichen und regelmäßigen Anwesenheit Gottes, es ist ein ,Präsenzmarker'. Mit dieser Theorie lässt sich eine Verhältnisbestimmung ausdrücken, die den Christen immer große Schwierigkeiten bereitet hat: Die Beziehung von Materiellem und Menschengestaltigem zum unsichtbaren Gott. Christus als den Materiellen und Menschengestaltigen zum Abbild und als identisch mit dem Urbild zu erklären, führte die antike Diskussion fort. Hier setzte die Bildtheologie an, und mit diesem solchermaßen gerechtfertigten Bild begann der Bilderkult in der christlichen Kirche. Auch dieses Bild erhielt ein Opfer, doch dessen blutiger Aspekt verschwand - durchaus im Einklang mit den religiösen Vorstellungen der Epoche - in einer Metapher. Es wurde aber als symbolische Handlung fortgeführt, und kein Gottesdienst verging ohne Beweihräucherung des Christusbildes.

\section{Kontinuitäten und Brüche in den Ritualen}

Ganz scharf haben die Christen zwei Aspekte der klassischen Kulte abgelehnt und zu dem Unterscheidungsmerkmal gegenüber ihrer Religion gemacht: Das Opfer und das Bild. Hinter einer solchen

${ }^{31}$ Die Perspektive von T. Mathews, The Clash of Gods (Princeton 1993) geht in die falsche Richtung mit der Annahme, dass die Neuheit und Schlagkraft der christlichen Bilder Ursache für den Untergang der paganen Götterbilder gewesen seien. Bei seiner expliziten Ablehnung der Verbindung des bärtigen Christusbildes mit dem Zeusbild kennt K.C. Felmy, Das Buch der Christus-Ikonen (Freiburg 2004), 12 weder das Material noch die historischen Hintergründe. 
ideologischen Zuspitzung eines Gegensatzes standen aber näher zu differenzierende Kontinuitäten und Brüche. Was die Christen bewusst aufgeben und auf die kaiserliche Autorität gestützt durch zahlreiche Verbote und Gesetze bekämpft haben, war das (blutige) Tieropfer und das dreidimensionale Götterbild. In beiden Abwehrbewegungen standen sie aber in der Kontinuität einer Entwicklung, die bereits lange zuvor eingesetzt hatte. Im christlichen Kult wurden andererseits viele Formen der klassischen Rituale fortgeführt, wohingegen die Kontinuität zu jüdischen Ritualen geringer ausfiel. Interessant ist etwa, wie das zentrale christliche Ritual der Eucharistie Elemente aufnahm, die unter Juden größten Abscheu ausgelöst haben müssen (so das Trinken von Blut) und sich am Modell des klassischen Opfers orientierten, allerdings nur in der Metapher. Manche der Rituale erfuhren dabei eine Differenzierung, um sich abzugrenzen. Viele aber wurden in der Weise ausgeübt, wie es die Städter im Imperium Romanum gewohnt waren. Es hatte sich somit eine Angleichung der ,Ritualkommunikation' durchgesetzt. ${ }^{32}$ Ohne die einzelnen Elemente noch einmal ausführlicher vorzustellen, sei hier nur kurz aufgeführt, welche ,kleinen` Opfer im christlichen Kult nun zur alltäglichen Gabe wurden: ${ }^{33}$

- Brot und Mehl

- Weihrauch

- Wachsopfer

- Licht und Kerzen

- Geld

Am Beispiel des Lichtes, des Entzündens von Öllampen und der relativ spezifischen Kerzen, lässt sich eine solche longue durée aufzeigen. Inwieweit es zu einer vermehrten Aktivität in diesem Bereich kam, ist schwer zu sagen, weil Statistiken über die Zahl der materiellen Zeugnisse für diese rituelle Praxis bislang nicht vorliegen. Der zufällige Erhaltungszustand archäologischer Befunde erlaubt noch keine Schlüsse über eine Zunahme (oder Abnahme) dieses Rituals. Aber es lassen sich Belege dafür finden, dass beispielsweise für das Lampenentzünden ein eigenes Amt eingeführt wurde, ohne dass dies einen religiösen Spezialisten voraussetzt:

\footnotetext{
${ }^{32}$ Vgl. das Schema am Ende dieses Beitrags.

${ }^{33}$ Ausführlicher bei Auffarth 2008a, a.a.O. (Anm. 1).
} 


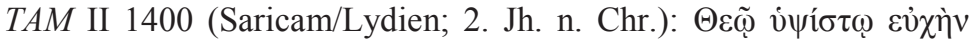

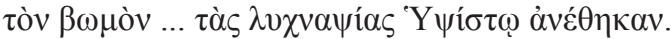

M.P. Nilsson, Eranos 54 (1956), 167-171 (Pergamon; kaiserzeitlich):

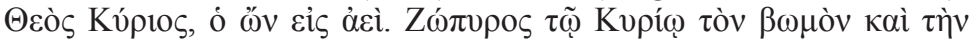

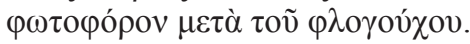

P.M. Petsas, Inscriptions du sanctuaire de la Mère des Dieux autochtone de Leukopetra (Paris 2000), Nr. 39 (Beroia/Makedonien;

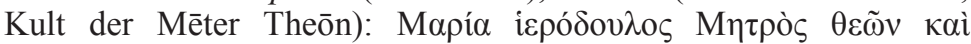
$\lambda v \chi v \alpha ́ \pi \tau \rho 1 \alpha$ (der Name Maria scheint semitischer Herkunft zu sein).

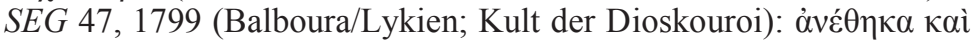

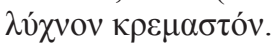

IGBulg III 1517 (Philippopolis/Thrakien; 3. Jh. n.Chr.; Kult des Dionysos): $\Lambda v \chi v o \alpha ́ \pi \tau \rho \alpha^{34}$

Das Götterbild wurde im Kult der Christen nicht mehr als dreidimensionales Kultbild verwendet, sondern bei der Aufnahme als ikonisches Verehrungsbild zweidimensional verkürzt. In Bezug auf das Ritual des Weihrauchopfers und der Weinspende am Bild kann man aber durchaus Kontinuitäten erkennen. Insbesondere im Ritual des Opfers vino ac ture ist der Anschluss an die rituelle Verehrung der imagines der Kaiser und ihrer Familie zu erkennen. Das rituelle Handeln veränderte sich im Laufe der Kaiserzeit, wobei die Ästhetisierung das Symbolhafte, die Individualisierung der Opfergabe in den Vordergrund treten lässt. Die Christen übernahmen und verstärkten diese längerfristigen Tendenzen. Da jetzt aber eine ,neue‘ Religion in Konkurrenz zu den klassischen Formen trat, stilisierten die Christen ihr kultisches Handeln als prinzipiell , anders', als Alternative, und leugneten die Kontinuität zu den bestehenden Kulten. Was als zeitgemäß-modern gelten sollte, wurde als christliche Neuerung definiert, was hingegen zu sehr traditionell geprägt war und den den alten Bräuchen anhing, wurde als hinterwäldlerisch-,pagan' gebrandmarkt. ${ }^{35}$

\footnotetext{
${ }^{34}$ Diese Belege verdanke ich Angelos Chaniotis; sie ließen sich leicht vermehren.

${ }^{35}$ J. Rüpke, 'Kult jenseits der Polisreligion: Polemiken und Perspektiven', Jahrbuch für Antike und Christentum 47 (2004), 5-15. Ders., in: C. Auffarth (Hrsg.): Religion auf dem Lande. Entstehung und Veränderung von Sakrallandschaften unter römischer Herrschaft (Stuttgart 2009), i.Dr.
} 


\section{Zusammenfassende Thesen}

Mein Beitrag kreist um die Frage, wie sich unter dem Einfluss der römischen Herrschaft die Rituale rund um das Götterbild veränderten. Dabei konnten folgende Beobachtungen gemacht werden:

- Die Kritik am Bild im Sinne der Verwechslung von Abbild und Vorbild ist allgemeine intellektuelle Überheblichkeit, die aber keine prinzipielle Kritik an der Einbeziehung der Bilder in das Ritual enthält. Die Differenz von Abbild und Vorbild ist eine alte Unterscheidung.

- Die Kritik spitzt sich besonders auf die theriomorphen Bilder der Ägypter zu und hat die Kanonisierung des anthropomorphen Götterbildes zur Folge, und hier wiederum der Meisterwerke der Klassik, die nun zahllos kopiert und auch in nicht-sakralen Räumen aufgestellt werden. Es handelt sich also um einen Vorgang der Kanonisierung und Vervielfältigung.

- Das Kunstwerk steht - Platons Kritik folgend - in der Konfrontation zwischen den unfähigen Künstlern, die im Materiellen verhaftet bleiben, und den schöpferischen Meistern, die die Materie lebendig zu machen verstehen. Die platonisierende Ästhetik führt demnach zu einer harmonischen Verwechslung von Kunst und Religion. Der Geschmack, der darüber entscheidet, wie Religion aussehen muss, orientiert sich an den wenigen unübertrefflichen Werken der Klassik: Diese Künstler bewirken die Epiphanie Gottes in dem idealen Bild, dessen Eigenschaften, obgleich materiell, Gottesidee und Gottesbild anschaulich werden lassen.

- Mit der Ästhetisierung der Religion lässt sich auch erklären, dass das Ritual des Tieropfers zurückgeht. Wiewohl es weiterhin unentbehrlich für große, vor allem öffentliche Akte bleibt, werden die anderen, ,kleinen' Opferformen zunehmend wichtiger. Hierfür werden nicht mehr bestimmte religiöse Spezialisten benötigt, und somit wird die Anwesenheit von Personen wie Metzgern, Wahrsagern oder Priestern entbehrlich.

-,Das Ende des Opfers' ist das Ende des blutigen Opfers. Die anderen, ,kleinen' Opfer werden weiterhin geübt und oft nur wenig verändert. Und diese kleinen Opfer bilden dann auch den Kern des christlichen Kultes.

- In den Ritualen zeigt sich somit eine ,Zivilisierung', die das blutige Opfer mehr und mehr zurückdrängt und die Ausübung ande- 
rer Opferformen wie des Entzündens von Lichtern, des Wohlgeruchs durch Weihrauch, des Mehlopfers und ähnlichem verstärkt. Damit verbunden ist, dass diese Formen auch ohne Opferpersonal und auch ohne festliche Gesellschaft ausgeübt werden können. Das bedeutet eine Individualisierung und Entprofessionalisierung, die die Kultausübung im Vorübergehen und im Alltag ohne den Aufwand eines Festes möglich macht.

- Als ab dem 4. Jh. eine christliche Semantik für die öffentlichen Plätze der Städten und ein monumentales Bild des verehrten Gottes in den Gotteshäusern benötigt wird, greifen die Christen die bereits vorhandene religiöse Ikonographie auf. Das musealisierte Sitzbild des Zeus von Olympia steht rund 50 Jahre lang in Konstantinopel, nicht mehr als religiöses Kultbild, sondern nun als ästhetisches Meisterwerk. Es wird zum Idealbild der Materie, in der sich dank der Schöpfung eines großen Künstlers die Anwesenheit des unsichtbaren Gottes erfahren lässt. Das platonische Modell vom Verhältnis zwischen dem göttlichen Urbild und dem materiellen Abbild wird zur Formel für die Christologie.

- Für die meisten Veränderungen in der Metamorphose der Religion gibt es einzelne Beispiele bereits aus klassisch-griechischer, zunehmend dann aus hellenistischer Zeit. Der Kaiserkult spiegelt diese Entwicklung, ist jedoch nicht Motor einer ,Mutation hin zur Loyalitätsreligion'.

- Zentral scheint mir die Ästhetisierung entlang eines griechischen Kanons zu sein. Diese Vereinheitlichung ist verknüpft mit einer Pluralisierung kultischer Vielfalt. Das Ganze aber lebt von der Kollaboration der hellenisierten Eliten mit den jeweiligen Machthabern. ${ }^{36}$

Bremen, Juni 2009

\footnotetext{
${ }^{36}$ Gegenüber den Bemerkungen von J. Hahn, 'Römische Herrschaft und Religion - Aspekte und Fragestellungen', in: ders. - L. de Blois - P. Funke (Hrsg.), The Impact of Imperial Rome on Religions, Ritual and Religious Life in the Roman Empire. Proceedings of the Fifth Workshop of the International Network Impact of Empire (Leiden - Boston 2006), 1-10 ergeben sich aus meiner Perspektive etwas andere Konsequenzen für Religion und Imperium: 1. Von Achaea aus gesehen hatte der militärische Aspekt eine geringere Bedeutung. 2. Offizielle Staatsreligion (Rituale zu Staatsakten) und persönliche Religion sind analytisch zu trennen, auch wenn sie sich m.E. gerade im Kult für die imagines der Kaiser wieder kreuzen, vgl. C. Auffarth, 'Herrscherkult und Christuskult', in: H. Cancik - K. Hitzl (Hrsg.), Die Praxis der Herrscherverehrung in Rom und seinen Provinzen (Tübingen 2003), 283-317.
} 


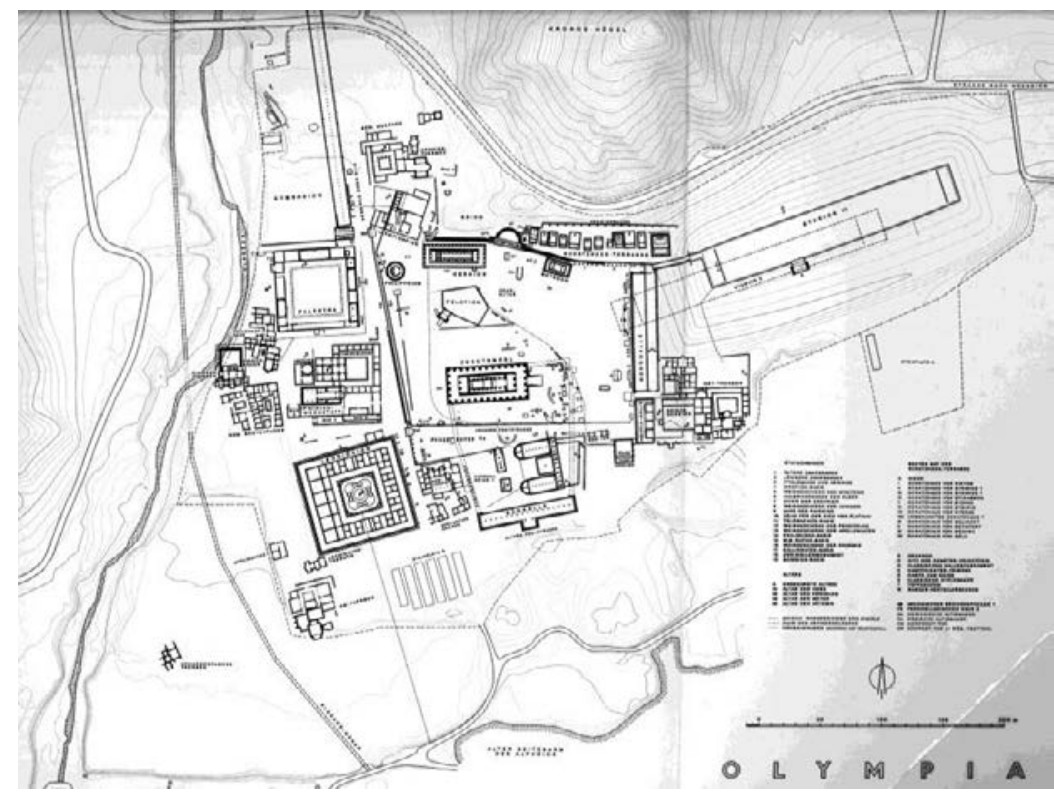

Abb. 1: Plan von Olympia: Die „Museums-Festung gegen die Heruler“. Aus: H. Kyrieleis (Hrsg.): Olympia 1875-2000 (Mainz 2002), Falttafel

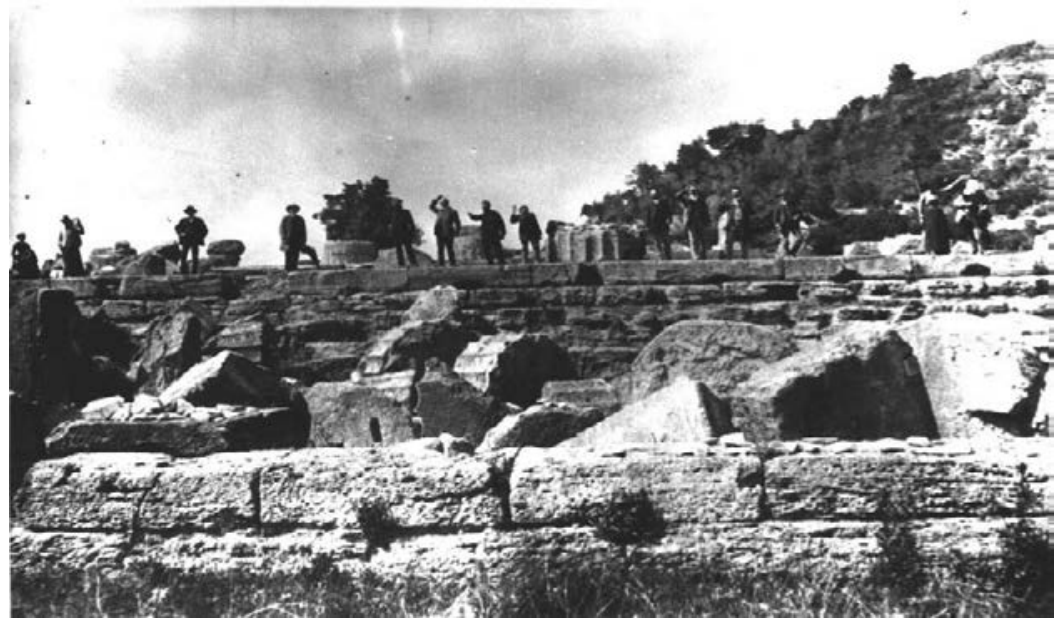

Abb. 2: Die Meisterwerke zwischen den Säulen (hier vertreten durch Mitglieder des Ausgrabungsteams). Photo: Archiv der Olympiagrabung, Neg. Nr. Olympia 148, Copyright Deutsches Archäologisches Institut Athen. 


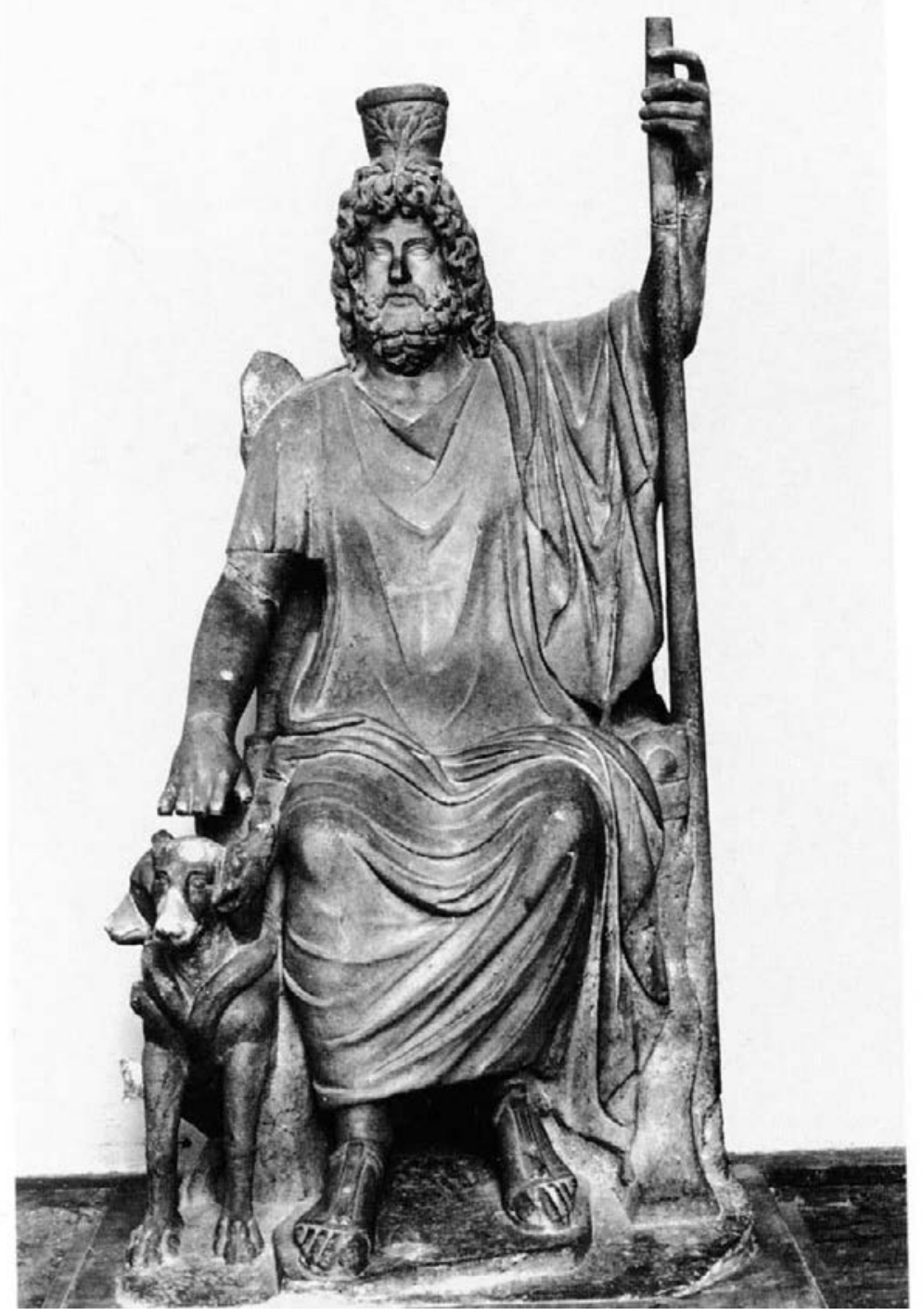

Abb. 3: Serapis in der Erscheinungsform des „,angemessenen Kultbildes“ des Zeus von Olympia: Serapis aus Puteoli (Dikaiarcheia), Nationalmuseum Neapel. Aus: R. Merkelbach, Isis Regina und Zeus Serapis (Stuttgart 1995), 591, Abb. 116. 


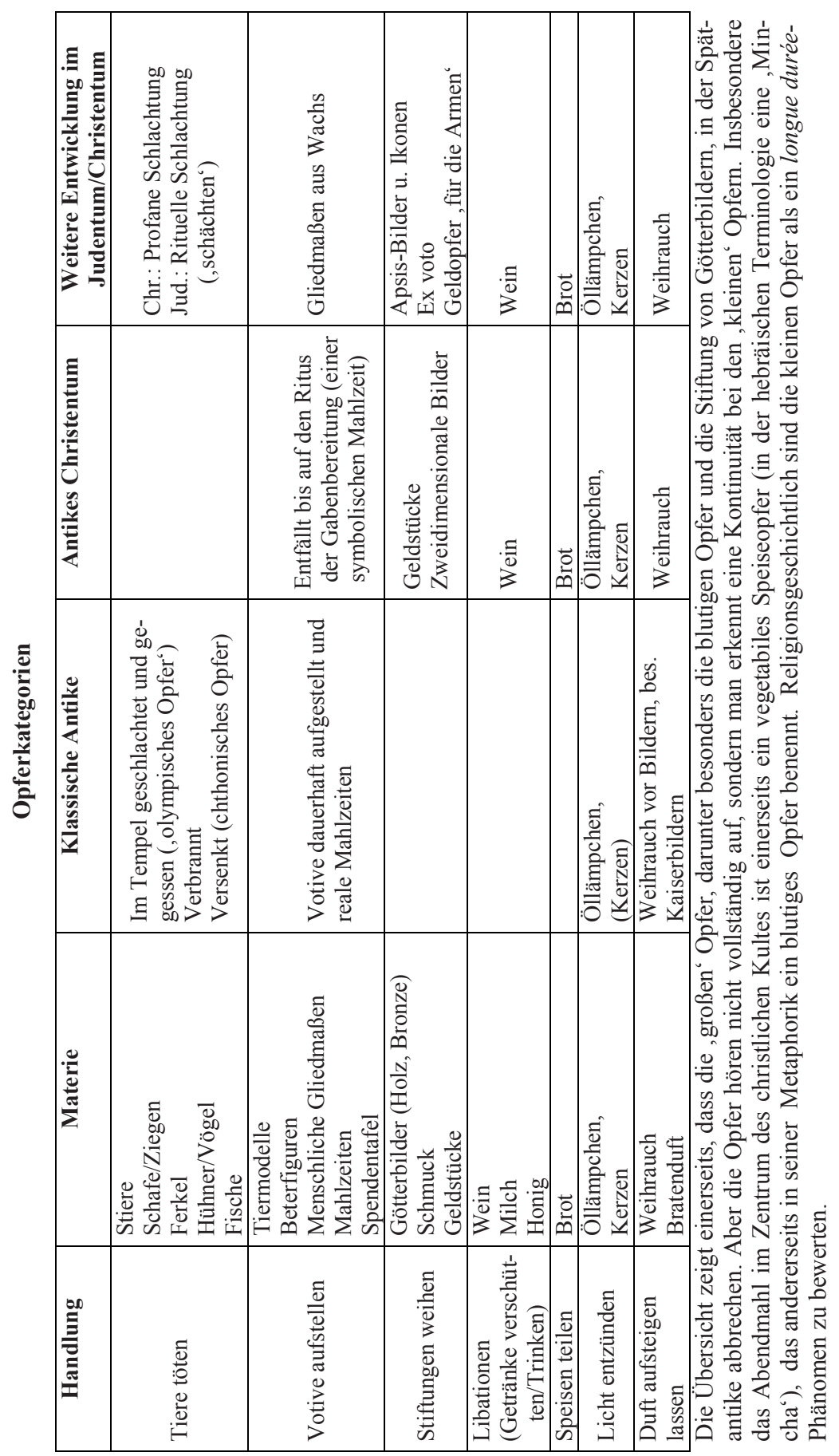

